\title{
Clinical Outcomes of Intra-arterial Chemoradiotherapy and Neoadjuvant Chemoradiotherapy Followed by Surgery for Maxillary Sinus Squamous Cell Carcinoma
}

\author{
Daisuke Yunaiyama ${ }^{1,{ }^{*}}$, Kiyoaki Tsukahara ${ }^{2}$, Mitsuro Okubo ${ }^{1}$ and Kazuhiro Saito ${ }^{1}$ \\ ${ }^{1}$ Department of Radiology, Tokyo Medical University, Shinjuku, Japan \\ ${ }^{2}$ Department of Otorhinolaryngology and Head and Neck Surgery, Tokyo Medical University, Shinjuku, Japan \\ "Corresponding author: Department of Radiology, Tokyo Medical University, Shinjuku, Japan. Email: yuunai1211@gmail.com
}

Received 2020 September 02; Revised 2021 August 16; Accepted 2021 August 28.

\begin{abstract}
Background: Although intra-arterial chemoradiotherapy (CRT) followed by surgery has been the standard of care for patients with advanced maxillary sinus squamous cell carcinoma (MSSCC), concurrent intra-arterial chemotherapy and high-dose radiotherapy without surgery has emerged as a promising alternative.

Objectives: This study aimed to evaluate the ability of intra-arterial CRT alone to increase the overall survival (OS) of patients with MSSCC.

Patients and Methods: Forty patients with histologically confirmed MSSCC, who were treated at Tokyo Medical University Hospital (Tokyo, Japan) between February 1999 and June 2015, were enrolled in this study. Twenty-seven patients were treated with intraarterial CRT (median dose of $60 \mathrm{~Gy}$ ) without surgery (CRT group), whereas 13 patients were treated with neoadjuvant intra-arterial CRT (median dose of $40 \mathrm{~Gy}$ ), followed by surgery (S group). The association of OS with age, performance status, T factor (mean tumor size according to the tumor-node-metastasis [TNM] grading system), $\mathrm{N}$ factor (mean lymph node involvement according to the TNM grading system), and treatment method was assessed.

Results: The median follow-up duration was 36.0 months. There were no significant differences regarding the patients' characteristics between the two groups. The treatment method was the only significant prognostic factor for OS. The five-year OS rates were 92\% and 55\% in the CRT and $S$ groups, respectively $(P=0.01)$.

Conclusion: The intra-arterial CRT (60 Gy) without surgery yielded improved survival outcomes in patients with advanced MSSCC as compared to the neoadjuvant intra-arterial CRT (40 Gy) followed by surgery.
\end{abstract}

Keywords: Maxillary Sinus Cancer, Squamous Cell Carcinoma, Chemoradiotherapy, Head and Neck Cancer

\section{Background}

Maxillary sinus carcinoma is the most common type of paranasal sinus cancer (1), and squamous cell carcinoma is the primary histological type of maxillary sinus tumors. Unlike other head and neck malignancies, maxillary sinus carcinomas are often diagnosed in locally advanced stages (2), owing to their localization and lack of symptoms in early stages. According to several studies, use of concurrent chemoradiotherapy can improve the locoregional control and overall survival (OS) of patients with locally advanced head and neck carcinomas, compared to radiotherapy alone (3-5).

Maxillary sinus tumors often advance locally without lymph node metastasis due to limited lymphatic drainage (6). Therefore, chemotherapy followed by surgical resec- tion, with or without radiotherapy, is the treatment of choice for patients with locally advanced maxillary sinus squamous cell carcinoma (MSSCC) (7-10). On the other hand, superselective cisplatin infusion with concomitant radiotherapy has emerged as a promising chemotherapeutic method (11-14).

\section{Objectives}

This study aimed to identify the optimal treatment regimen for patients with MSSCC by retrospectively reviewing the outcomes of MSSCC patients, who were treated with neoadjuvant intra-arterial chemoradiotherapy (NACR) followed by surgery, as well as patients who received definitive intra-arterial chemoradiotherapy alone. 


\section{Patients and Methods}

This retrospective study was approved by the institutional review board (approval code: T2019-0262), and the requirement for written informed consent was waived.

\subsection{Study Population}

A total of 49 consecutive patients with histologically confirmed MSSCC, who were treated at Tokyo Medical University Hospital (Tokyo, Japan) between February 1999 and June 2015, were examined in this study. Nine patients who were treated with intravenous chemoradiotherapy or radiotherapy alone were excluded from the study due to impaired renal function or poor performance, according to the definition by the Eastern Cooperative Oncology Group (ECOG) (15). Finally, 27 out of the remaining 40 patients were treated with intra-arterial chemoradiotherapy alone at a median dose of $60 \mathrm{~Gy}$ (CRT group), whereas 13 patients were treated with intra-arterial chemoradiotherapy at a median dose of $40 \mathrm{~Gy}$, followed by surgery (S group).

The available treatment methods varied in the present study, because the time frame of the study was more than a decade, which could influence the therapeutic options. However, there were no significant differences between the two groups in terms of age, sex, tumor-node-metastasis (TNM) grading ( $8^{\text {th }}$ edition by the Union for International Cancer Control [UICC]), tumor stage, or performance status (Table 1).

Over the course of this study, many advances were made in the available treatment methods, chemotherapy regimens, and irradiation methods (Table 2). Although cisplatin-based compounds were recognized as the chemotherapy agents of choice for both groups of patients, various regimens without cisplatin were used in early studies, particularly for the $S$ group of patients (Table 3).

This retrospective study was conducted according to the World Medical Association Declaration of Helsinki and was approved by the institutional review board. The requirement for informed consent was waived in this study.

\subsection{Treatments}

In the CRT group $(\mathrm{n}=27)$, chemotherapeutic agents were administered via intra-arterial infusion using a superselective catheter for 21 patients, whereas for six patients, a retrograde trans-superficial temporal arterial infusion was performed using an arterial infusion port system. In the $S$ group, chemotherapeutic agents were administered via intra-arterial infusion for nine out of 13 patients, while the remaining four patients were treated with retrograde trans-arterial infusion chemotherapy.

\begin{tabular}{|c|c|c|c|}
\hline & CRT group $(n=27)$ & $S$ group $(n=13)$ & P-value \\
\hline Median age $(y)$ & $61(40-86)$ & $61(45-79)$ & 0.65 \\
\hline Sex & & & 0.89 \\
\hline Male & 16 & 8 & \\
\hline Female & 11 & 5 & \\
\hline T classification & & & 0.62 \\
\hline $\mathrm{T} 2$ & 2 & 2 & \\
\hline $\mathrm{T} 3$ & 5 & 4 & \\
\hline T4a & 16 & 6 & \\
\hline $\mathrm{T} 4 \mathrm{~b}$ & 4 & 1 & \\
\hline $\mathrm{N}$ classification & & & 0.65 \\
\hline No & 23 & 12 & \\
\hline N1 & 1 & 0 & \\
\hline N2 & 3 & 1 & \\
\hline N3 & 0 & 0 & \\
\hline M classification & & & NA \\
\hline Mo & 27 & 13 & \\
\hline Stage & & & 0.755 \\
\hline II & 2 & 2 & \\
\hline III & 6 & 4 & \\
\hline IVA & 16 & 6 & \\
\hline IVB & 3 & 1 & \\
\hline Performance status & & & 0.96 \\
\hline 0 & 23 & 11 & \\
\hline 1 & 4 & 2 & \\
\hline
\end{tabular}

Abbreviations: CRT, chemoradiotherapy, S, chemoradiotherapy followed by surgery, NA, not applicable; T, tumor; N, node; M, metastasis.

Intra-arterial chemotherapy was performed by a radiologist. During this period, three other radiologists participated in the therapeutic procedures under the radiologist's guidance. Intra-arterial chemotherapy involved the administration of high doses of cisplatin $\left(200 \mathrm{mg} / \mathrm{m}^{2}\right)$ using angiography-assisted computed tomography (CT) to allow for whole tumor coverage, as previously reported (16). The intra-arterial infusion of chemotherapeutic agents through the superficial temporal artery was performed according to the protocol proposed by Robins et al. (13). The patients in the CRT group received chemotherapy on days 1 and 36 , while the $S$ group received chemotherapy on day 1 . The patients in the CRT group were irradiated at a median dose of $60 \mathrm{~Gy}$, whereas the $S$ group received a median dose of $40 \mathrm{~Gy}$, followed by surgery. Surgical margins were defined based on the tumor bed before NACR. 


\begin{tabular}{lcc}
\hline Table 2. The Treatment Period and Irradiation Doses & & \\
\hline & CRT group $(\mathbf{n}=\mathbf{2 7})$ & S group $(\mathbf{n}=\mathbf{1 3})$ \\
\hline Treatment period & October 2000 - May 2015 & February 1999- February 2008 \\
Median irradiated dose (Gy) & $60(26-70)$ & $40(34-60)$ \\
\hline
\end{tabular}

Abbreviations: CRT, chemoradiotherapy; S, chemoradiotherapy followed by surgery.

\begin{tabular}{lccc}
\hline Table 3. The Regimens in the CRT and S Groups & & \\
\hline & CRT group & S group & P-value \\
\hline CDDP & 6 & 3 & 0.06 \\
CDDP + 5-FU & 13 & 2 & \\
CDDP + DOC & 3 & 2 & \\
CDDP + 5-FU + DOC & 1 & & \\
CDDP + DOC + TS-1 & 1 & & \\
CDDP + 5-FU + UFT & 1 & 6 \\
CDDP + ADM + UFT & 1 & 1 \\
ADM & & \\
\hline
\end{tabular}

Abbreviations: CRT, chemoradiotherapy; S, chemoradiotherapy followed by surgery; CDDP, cisplatin; 5FU, 5-fluorouracil; DOC, docetaxel; TS-1, tegafurgimeracil-oteracil potassium; UFT, tegafur-uracil; ADM, adriamycin.

\subsection{Evaluations}

Tumor response was evaluated by contrast-enhanced CT scan, magnetic resonance imaging (MRI), and endoscopy at two months after the end of treatment, according to the new Response Evaluation Criteria in Solid Tumors (RECIST) criteria (revised RECIST guideline, version 1.1) (17). Along with response evaluation according to the revised RECIST criteria, biopsy was performed for the patients.

The primary endpoint of this study was the OS measurement, and the secondary endpoint was the assessment of progression-free survival (PFS), both of which were calculated from the first day of treatment. The PFS was defined as the time interval from the first day of the therapeutic procedure until the time of death documented in the medical record or recurrence confirmed by CT or MRI. Besides, adverse events during treatment were defined according to the Common Terminology Criteria for Adverse Events (CTCAE) version 4 (18).

\subsection{Statistical Analysis}

All statistical analyses were performed in SPSS version 27.0 (IBM Corporation, Armonk, NY, USA). A P-value less than 0.05 was considered statistically significant. The OS and PFS rates were plotted for patients in the CRT and $S$ groups, using the Kaplan-Meier method, and the survival curves were compared based on the log-rank test. The prognostic factors for OS and PFS were identified in a multivariate analysis, using the Cox proportional-hazards model. The incidence of adverse events was also examined in the CRT and $S$ groups by Chi-square test ( $\chi^{2}$ test).

\section{Results}

The median follow-up duration was 36.0 months (range: 13 - 45 months) in the patients. Also, the median follow-up duration of patients in the CRT and $S$ groups was 27.0 months (range: 2 - 142 months) and 43.0 months (range: 2 -194 months), respectively. All patients received a complete treatment. The five-year OS rates were $94.7 \%$ and $51.9 \%$ in the CRT and S groups, respectively, and the difference between the groups was significant $(\mathrm{P}<0.01)$ (Figure 1). Also, the five-year PFS rates of patients in the CRT and $S$ groups were estimated at $65.8 \%$ and $57.5 \%$, respectively $(\mathrm{P}=$ 0.917) (Figure 2).

During the follow-up, five patients in the $S$ group were diagnosed with local recurrence and expired in a median duration of ten months after the second-line therapy (Figure 3). Three patients in the CRT group were also diagnosed with tumor recurrence; two of them survived after surgery as a second-line treatment (Figure 4), while one patient died after receiving the best supportive care. The characteristics and outcomes of patients with tumor recurrence are presented in Table 4. There was no significant difference in the five-year PFS rates between the patients in the CRT and S groups (70\% vs. $61 \% ; \mathrm{P}=0.78$ ).

Based on the multivariate analysis using a Cox proportional-hazards model, among all variables, the treatment method was the only significant prognostic factor $(P=0.01$ ) (Table 5). Regarding the development of treatment-related adverse effects, grade 2 toxicity occurred in five out of 27 patients in the CRT group, while such adverse effects were observed in two out of 13 patients in the $S$ group. Besides, grade 3 toxicity occurred in five patients in the CRT group and two patients in the $S$ group (Table 6). Nevertheless, there was no significant difference between the two groups regarding grade 2 or grade 3 toxicity $(\mathrm{P}=0.424$ and $\mathrm{P}=0.773$, respectively). 


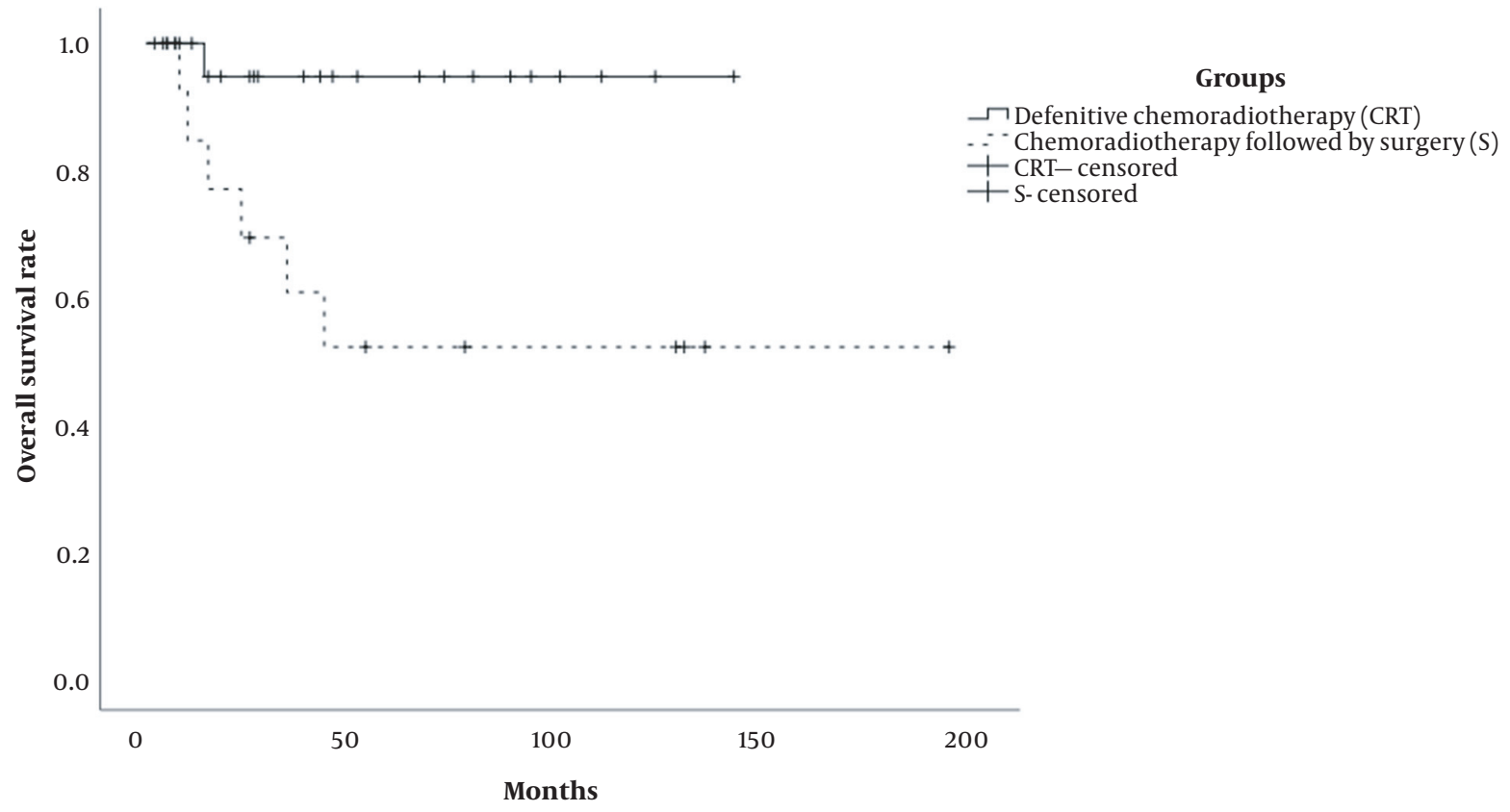

Figure 1. The overall survival (OS) of patients treated with chemoradiotherapy alone and patients treated with chemoradiotherapy followed by surgery.

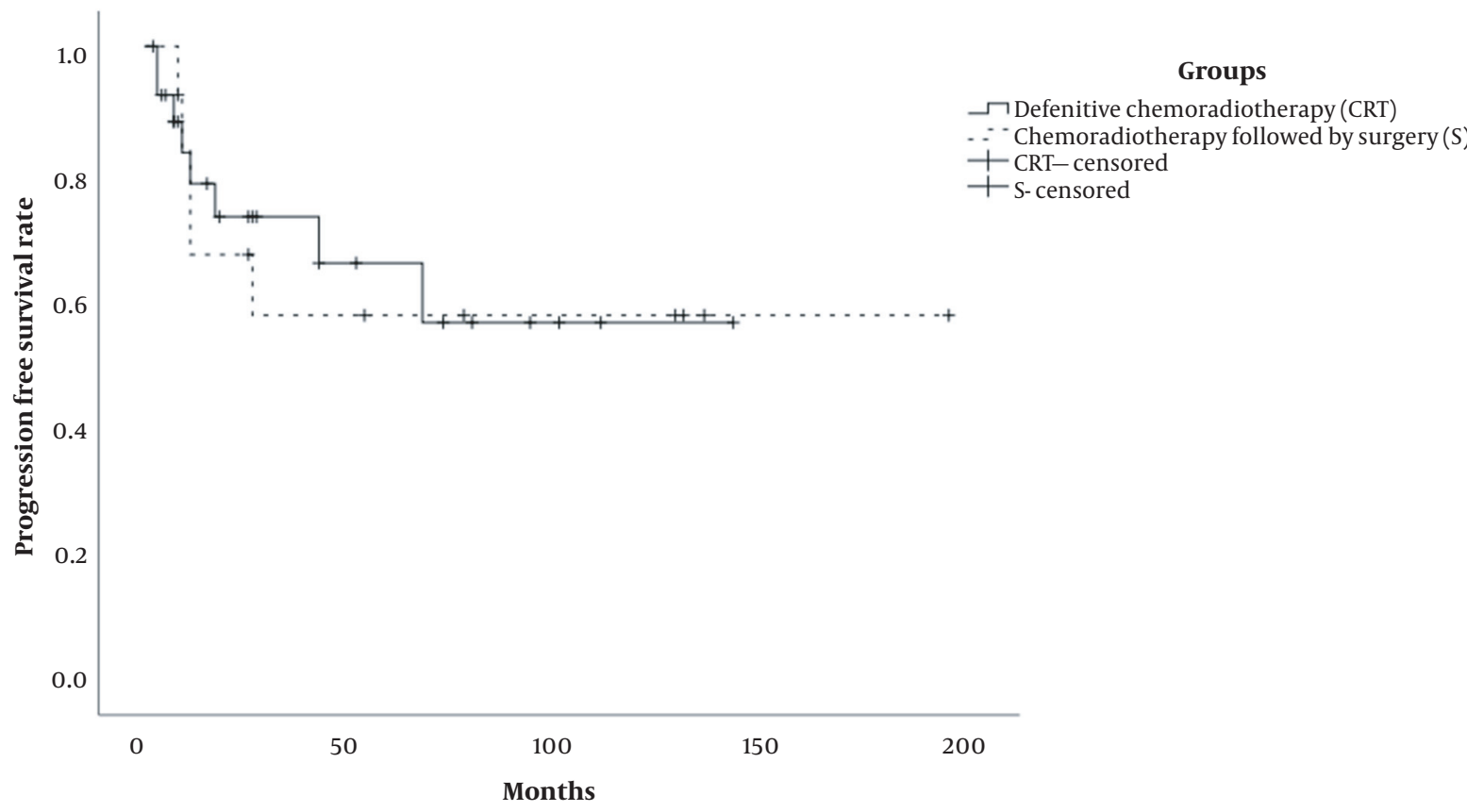

Figure 2. The progression-free survival (PFS) of patients treated with chemoradiotherapy alone and patients treated with chemoradiotherapy followed by surgery. 

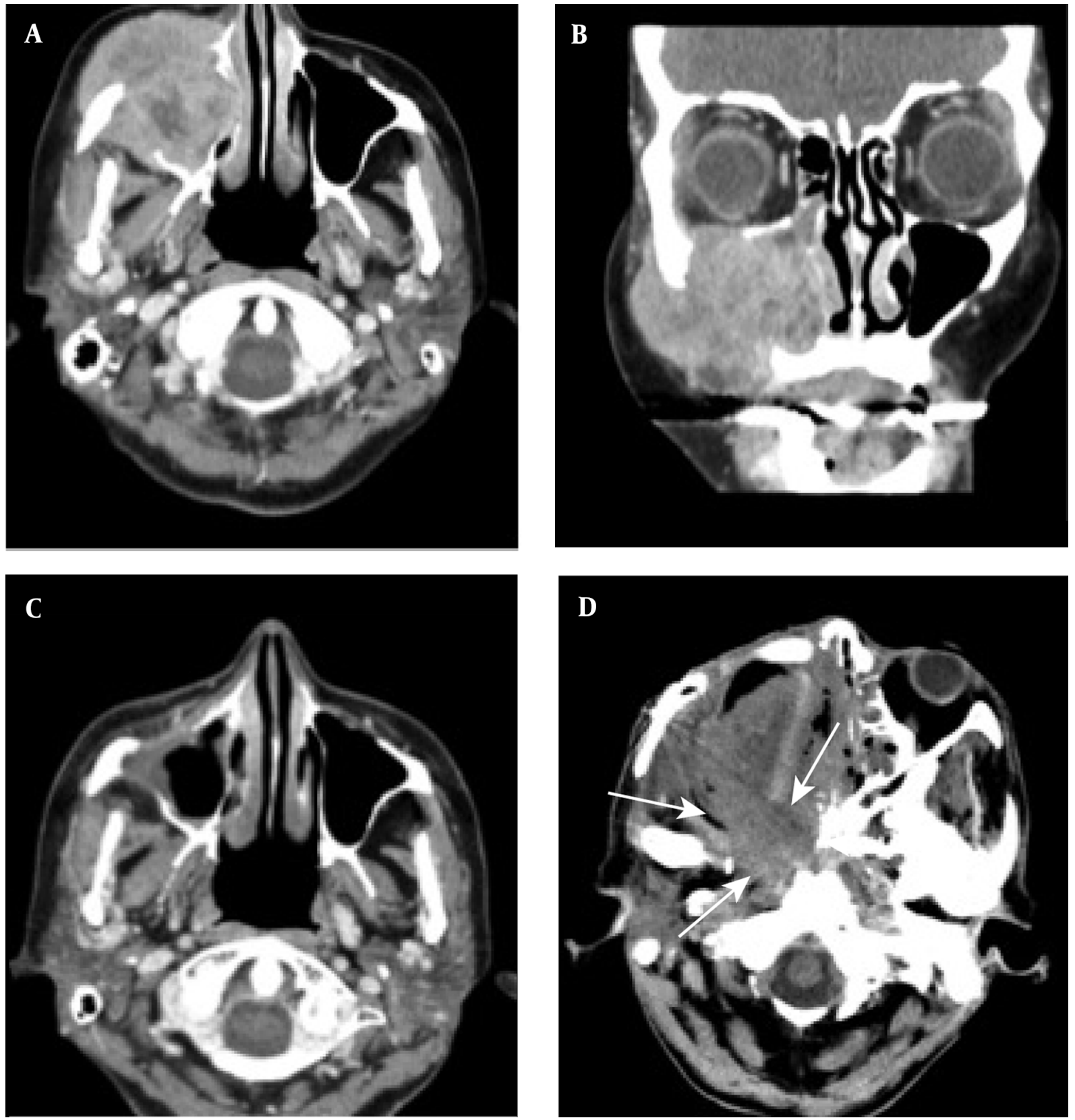

Figure 3. A case of locally advanced maxillary sinus carcinoma treated with chemoradiotherapy followed by surgery. A, Axial contrast-enhanced CT scan shows a large mass in the maxillary sinus on the right side, invading into the subcutaneous tissue. B, Coronal contrast-enhanced CT scan shows tumor invasion into the orbital space on the right side. C, Axial contrast-enhanced CT scan shows tumor remission. D, Axial contrast-enhanced CT scan indicates tumor recurrence (arrows). The patient received the best supportive care, but expired due to tumor progression.

\section{Discussion}

In the present study, the treatment method was recognized as a prognostic factor in patients with MSSCC, while treatment-related adverse effects were similar in the two groups. The CRT group underwent superselective and ret- rograde chemotherapy. An angiography-assisted CT scan could confirm that the entire tumor volume was covered in both methods of chemotherapy. To discuss differences between the groups, the infusion speed was compared. However, since there was no detailed information in the patients' medical records, the next step was to validate 

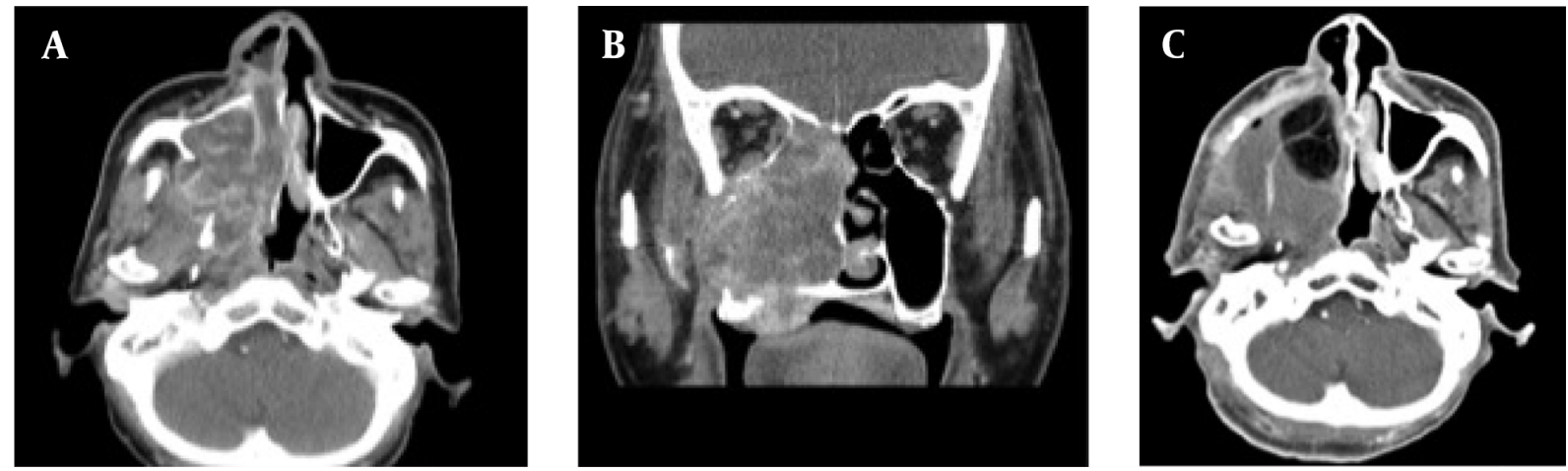

Figure 4. A case of locally advanced maxillary sinus carcinoma treated with definitive chemoradiotherapy. A, Axial contrast-enhanced CT scan shows a large mass in the maxillary sinus on the right side, invading into the lateral pterygoid muscle, pterygoid plate, and tensor veli palatini muscle. B, Coronal contrast-enhanced CT scan shows tumor invasion into the buccal space, ethmoid sinus, and hard palate. C, Axial contrast-enhanced CT scan shows a residual tumor after definitive chemoradiotherapy.

\begin{tabular}{|c|c|c|c|}
\hline Time to recurrence (mo) & Treatment methods & Outcomes & $\begin{array}{c}\text { Observation period after } \\
\text { additional treatment (mo) }\end{array}$ \\
\hline \multicolumn{4}{|c|}{ S group } \\
\hline 35 & Arterial chemotherapy & Expired & 10 \\
\hline 3 & Neck dissection & Expired & 7 \\
\hline 13 & Subtotal maxillectomy & Expired & 7 \\
\hline 4 & Chemoradiotherapy & Expired & 11 \\
\hline 7 & BSC & Expired & 27 \\
\hline \multicolumn{4}{|c|}{ CRT group } \\
\hline 2 & Subtotal maxillectomy & Alive & 24 \\
\hline 17 & Partial resection & Alive & 27 \\
\hline 13 & BSC & Expired & 1 \\
\hline
\end{tabular}

Abbreviations, BSC, best supportive care, S, surgery, CRT, chemoradiotherapy.

\begin{tabular}{lccc}
\hline Table 5. The Cox Proportional-Hazards Model & & \\
\hline Variables & \multicolumn{4}{l}{ Hazard ratio $(95 \%$ CI $)$} & P-value \\
\hline Age, $>$ 60 years vs. $<$ 60 years & 3.92 & $(0.624-24.68)$ & 0.15 \\
$\begin{array}{l}\text { Sex, male vs. female } \\
\text { Performance status }\end{array}$ & 1.86 & $(0.243-14.26)$ & 0.55 \\
$\begin{array}{l}\text { T classification, } \geq \text { T4 vs. T2 and T3 } \\
\text { N classification, positive vs. }\end{array}$ & 2.45 & $(0.380-15.79)$ & 0.35 \\
negative & 0.24 & $(0.051-3.472)$ & 0.30 \\
\begin{tabular}{l} 
Treatment method, CRT vs. $S$ \\
\hline
\end{tabular} & 0.05 & $(0.004-0.538)$ & 0.01 \\
\hline
\end{tabular}

Abbreviations: CRT, chemoradiotherapy, S, chemoradiotherapy followed by surgery, CI, confidence interval.

the infusion speed in trans-arterial administrations. Five and three patients relapsed in the CRT and S groups, respectively. All five patients with tumor recurrence in the
$S$ group expired despite additional second-line therapies. In contrast, two out of three patients with relapse in the CRT group survived after surgical resection. Therefore, intra-arterial chemoradiotherapy was proposed as a definite treatment for MSSCC, providing improved outcomes as compared to chemoradiotherapy followed by surgery.

Although we cannot exclude the possibility of selection bias, the patients exhibited improved five-year OS and PFS rates. In this regard, Nishimura et al. (19) reported a five-year OS rate of $59.2 \%$ in 40 patients with locally advanced MSSCC treated with CRT. Similarly, Hayashi et al. (8) reported a five-year OS rate of $68.5 \%$ and a local control (LC) rate of $84 \%$ in 62 MSSCC patients, treated with multimodality therapy. Additionally, Nibu et al. (20) reported a five-year OS rate of 74\% in 33 MSSCC patients treated with preoperative chemoradiotherapy, surgery, and postoperative radiotherapy. The five-year OS rate reported in the 


\begin{tabular}{|c|c|c|c|c|}
\hline & \multicolumn{2}{|c|}{ CRT group } & \multicolumn{2}{|c|}{ Sgroup } \\
\hline & Grade 2 & Grade 3 & Grade 2 & Grade 3 \\
\hline Infection & 1 & 1 & & \\
\hline Mucositis & & 1 & & \\
\hline Dysphagia & & 1 & & \\
\hline Nausea & 1 & & & \\
\hline Loss of appetite & & & & 1 \\
\hline Dermatitis & & & 1 & \\
\hline Pulpitis & 1 & & & \\
\hline Elevated liver enzymes & 1 & & & \\
\hline Renal failure & 1 & & & \\
\hline Osteonecrosis & & 1 & & 1 \\
\hline Facial nerve disorder & & 1 & & \\
\hline Maxillary nerve disorder & & & 1 & \\
\hline
\end{tabular}

Abbreviations: CTCAE, common terminology criteria for adverse events, CRT, chemoradiotherapy, S, chemoradiotherapy followed by surgery.

present study (93\%) suggests that CRT has more clinical advantages than treatment methods used in previous studies.

To increase the intensity of therapy locally for MSSCC patients, several researchers have used high-dose intraarterial chemoradiotherapy. In this regard, Kaneko et al. (21) reported that intra-arterial chemoradiotherapy with weekly low-dose cisplatin led to a five-year LC rate of $63.0 \%$ and a five-year OS rate of $75 \%$ in MSSCC patients. Similarly, Yoshimura et al. (22) reported a five-year OS rate of $63 \%$ in 110 MSSCC patients treated with radiotherapy, intraarterial chemotherapy, and antrotomy.

Moreover, Homma et al. (23) showed that the five-year local progression survival and five-year OS rates were $65.8 \%$ and $67.9 \%$ in MSSCC patients treated with intra-arterial cisplatin infusion and concomitant radiotherapy, respectively. They also conducted a dose-escalation study to determine the optimal dose of cisplatin for intra-arterial chemoradiotherapy (24). In the present study, intraarterial infusion using a superselective catheter allowed for the administration of a high dose of cisplatin. Besides, the use of CT-assisted monitoring ensured the coverage of the entire tumor, contributing to improved clinical outcomes using CRT. Overall, surgical interventions were significantly associated with a poor prognosis.

Several studies have demonstrated that nodal involvement is not a significant predictive factor for neck cancer relapse $(25,26)$. Consistently, in the current study, nodal involvement was not a significant predictive factor for recurrence. Considering the limited lymph drainage through the emissary veins, neck lymph node involvement can sug- gest high tumor invasiveness. Further studies are required to elucidate the significance of nodal involvement in the recurrence of MSSCC.

In the present study, no patients treated with CRT alone developed adverse effects (grade 4 toxicity or higher). In this regard, Rasch et al. (14) reported that the frequency of grade 2 renal toxicity (or higher) was significantly lower in patients treated with intra-arterial CRT. One patient suffered from grade 2 renal dysfunction, which was temporary. Also, one patient in the CRT group developed facial nerve palsy after chemoinfusion. Moreover, Sugiyama et al. (27) showed that cisplatin infusion into the middle meningeal artery (MMA) resulted in facial nerve palsy, because the feeding artery of the facial nerve arises from the petrosal branch of MMA. In the current study, to minimize the likelihood of facial nerve palsy, the chemotherapeutic agents were infused in the second portion of the maxillary artery, as maxillary sinus carcinoma extended to the lateral pterygoid muscle. However, this adverse event is limited to superselective chemotherapy, and it is preventable with an accurate knowledge of MMA anatomy.

The main limitations of this study were the retrospective design and several possible biases. To deal with the effect of bias, statistical analysis was performed using a Cox proportional-hazards model, which is a multivariate analysis. Moreover, the baseline characteristics of the patients could vary depending on many factors, such as chemotherapeutic agents and intra-arterial chemotherapy techniques. However, this study revealed that the therapeutic transition was not distressing for the patients, because the OS rate improved based on the comparison of 
the CRT and S groups. Finally, despite the ability of superselective CRT to improve the outcomes, the superselective intra-arterial infusion of chemotherapeutic agents is technically challenging; this problem could be overcome by the appropriate technical training of physicians and the improvement of catheters and guidewires. Overall, a multi-institutional phase III trial is needed to demonstrate the effectiveness of intra-arterial chemotherapy in patients with advanced MSSCC.

In conclusion, patients in the CRT group showed significantly prolonged $O S$ rates compared to the $S$ group. The definitive intra-arterial chemoradiotherapy increased the OS of patients with advanced MSSCC, based on a multivariate analysis. In the future, we will conduct a phase II clinical trial for a comprehensive comparison of CRT and $S$ groups.

\section{Acknowledgments}

The authors would like to thank the Editage (www.editage.com) for their English language editing services.

\section{Footnotes}

Authors' Contribution: Study concept and design: D.Y. and K.T.; Acquisition of data: D.Y.; Analysis and interpretation of data: D.Y. and K.S.; Drafting of the manuscript: D.Y.; Critical revision of the manuscript for important intellectual content: D.Y., K.S., M.O., and K.T.; Statistical analysis: D.Y. and K.T.; administrative, technical, and material support: M.O. and K.S.; And study supervision: K.T.

\section{Conflict of Interests: None.}

\section{Ethical Approval: T2019-0262.}

Funding/Support: The authors declare that they have received no funding support.

\section{References}

1. Ayiomamitis A, Parker L, Havas T. The epidemiology of malignant neoplasms of the nasal cavities, the paranasal sinuses and the middle ear in Canada. Arch Otorhinolaryngol. 1988;244(6):367-71. doi: 10.1007/BF00497467. [PubMed: 3279940].

2. Paulino AC, Marks JE, Bricker P, Melian E, Reddy SP, Emami B. Results of treatment of patients with maxillary sinus carcinoma. Cancer. 1998;83(3):457-65. doi: 10.1002/(sici)10970142(19980801)83:3<457::Aid-cncr14>3.0.Co;2-v.

3. Pignon JP, Bourhis J, Domenge C, Designé L. Chemotherapy added to locoregional treatment for head and neck squamous-cell carcinoma: Three meta-analyses of updated individual data. Lancet. 2000;355(9208):949-55. doi:10.1016/s0140-6736(00)90011-4.
4. Wendt TG, Grabenbauer GG, Rodel CM, Thiel HJ, Aydin H, Rohloff R, et al. Simultaneous radiochemotherapy versus radiotherapy alone in advanced head and neck cancer: A randomized multicenter study. $J$ Clin Oncol.1998;16(4):1318-24. doi:10.1200/JCO.1998.16.4.1318. [PubMed: 9552032].

5. Browman GP, Hodson DI, Mackenzie RJ, Bestic N, Zuraw L, Cancer Care Ontario Practice Guideline Initiative $\mathrm{H}$, et al. Choosing a concomitant chemotherapy and radiotherapy regimen for squamous cell head and neck cancer: A systematic review of the published literature with subgroup analysis. Head Neck. 2001;23(7):579-89. doi: 10.1002/hed.1081. [PubMed: 11400247].

6. Dooley L, Shah J. Management of the neck in maxillary sinus carcinomas. Curr Opin Otolaryngol Head Neck Surg. 2015;23(2):107-14. doi: 10.1097/MOO.0000000000000138. [PubMed: 25692625]. [PubMed Central: PMC4968034].

7. Kang JH, Cho SH, Kim JP, Kang KM, Cho KS, Kim W, et al. Treatment outcomes between concurrent chemoradiotherapy and combination of surgery, radiotherapy, and/or chemotherapy in stage III and IV maxillary sinus cancer: multi-institutional retrospective analysis. $J$ Oral Maxillofac Surg. 2012;70(7):1717-23. doi: 10.1016/j.joms.2011.06.221. [PubMed: 21945430].

8. Hayashi $\mathrm{T}$, Nonaka S, Bandoh $\mathrm{N}$, Kobayashi $\mathrm{Y}$, Imada $\mathrm{M}$, Harabuchi Y. Treatment outcome of maxillary sinus squamous cell carcinoma. Cancer. 2001;92(6):1495-503. doi: 10.1002/10970142(20010915)92:6<1495::Aid-cncr1474>3.0.Co;2-p.

9. Dulguerov P, Jacobsen MS, Allal AS, Lehmann W, Calcaterra T. Nasal and paranasal sinus carcinoma: Are we making progress? Cancer. 2001;92(12):3012-29. doi: 10.1002/1097-0142(20011215)92:12<3012::Aidcncr10131>3.0.Co;2-e.

10. National Compewhwnsive Cancer Network. Oncology NCPGi. 2010 Available from: http://www.nccn.org/professionals/physician_gls/ f_guidelines.asp..

11. Kumar P, Robbins KT. Treatment of advanced head and neck cancer with intra-arterial cisplatin and concurrent radiation therapy: the 'RADPLAT' protocol. Curr Oncol Rep. 2001;3(1):59-65. doi: 10.1007/s11912001-0044-1. [PubMed: 11123871].

12. Robbins KT, Homma A. Intra-arterial chemotherapy for head and neck cancer: experiences from three continents. Surg Oncol Clin N Am. 2008;17(4):919-33. xi. doi: 10.1016/j.soc.2008.04.015. [PubMed: 18722926].

13. Robbins KT, Kumar P, Harris J, McCulloch T, Cmelak A, Sofferman R, et al. Supradose intra-arterial cisplatin and concurrent radiation therapy for the treatment of stage IV head and neck squamous cell carcinoma is feasible and efficacious in a multi-institutional setting: results of Radiation Therapy Oncology Group Trial 9615. J Clin Oncol. 2005;23(7):1447-54. doi:10.1200/JCO.2005.03.168. [PubMed: 15735120].

14. Rasch CR, Hauptmann M, Schornagel J, Wijers O, Buter J, Gregor T, et al. Intra-arterial versus intravenous chemoradiation for advanced head and neck cancer: Results of a randomized phase 3 trial. Cancer. 2010;116(9):2159-65. doi: 10.1002/cncr.24916. [PubMed: 20187094].

15. Oken MM, Creech RH, Tormey DC, Horton J, Davis TE, McFadden ET, et al. Toxicity and response criteria of the Eastern Cooperative Oncology Group. Am J Clin Oncol.1982;5(6):649-55. [PubMed: 7165009].

16. Yunaiyama D, Saito K, Funatsu T, Nakayama H, Shimizu A, Ito H, et al. Effectiveness of CT assists for intraarterial chemotherapy: therapeutic outcome of chemoradiation for advanced head and neck cancer extending across the anatomical midline.Jpn JRadiol.2014;32(12):70815. doi: 10.1007/s11604-014-0370-5. [PubMed: 25380786].

17. Eisenhauer EA, Therasse P, Bogaerts J, Schwartz LH, Sargent D, Ford $\mathrm{R}$, et al. New response evaluation criteria in solid tumours: revised RECIST guideline (version 1.1). Eur J Cancer. 2009;45(2):228-47. doi: 10.1016/j.ejca.2008.10.026. [PubMed:19097774].

18. Nationa Cancer Institute. Common terminology criteria for adverse events (ctcae) version 4.0. 2010. Available from: https: //ctep.cancer.gov/protocoldevelopment/electronic_applications/ docs/ctcae_4_with_lay_terms.pdf. 
19. Nishimura G, Tsukuda M, Mikami Y, Matsuda H, Horiuchi C, Satake $\mathrm{K}$, et al. The efficacy and safety of concurrent chemoradiotherapy for maxillary sinus squamous cell carcinoma patients. Auris Nasus Larynx. 2009;36(5):547-54. doi:10.1016/j.anl.2008.11.002. [PubMed: 19097833].

20. Nibu K, Sugasawa M, Asai M, Ichimura K, Mochiki M, Terahara A, et al. Results of multimodality therapy for squamous cell carcinoma of maxillary sinus. Cancer. 2002;94(5):1476-82. doi: 10.1002/cncr.10253. [PubMed: 11920504].

21. Kaneko T, Tada Y, Maruya S, Takeishi E, Miura K, Masubuchi T, et al. Intra-arterial chemoradiation therapy with weekly low-dose cisplatin for squamous cell carcinoma of the maxillary sinus. Int J Oral Maxillofac Surg. 2015;44(6):697-704. doi: 10.1016/j.ijom.2015.03.009. [PubMed: 25843537].

22. Yoshimura R, Shibuya H, Ogura I, Miura M, Amagasa T, Enomoto S, et al. Trimodal combination therapy for maxillary sinus carcinoma. Int J Radiat Oncol Biol Phys. 2002;53(3):656-63. doi: 10.1016/s03603016(02)02771-2.

23. Homma A, Sakashita T, Yoshida D, Onimaru R, Tsuchiya K, Suzuki $\mathrm{F}$, et al. Superselective intra-arterial cisplatin infusion and concomitant radiotherapy for maxillary sinus cancer. $\mathrm{Br} J$ Cancer.
2013;109(12):2980-6. doi: 10.1038/bjc.2013.663. [PubMed: 24196792]. [PubMed Central: PMC3859946].

24. Homma A, Nakamura K, Matsuura K, Mizusawa J, Onimaru R, Fukuda $\mathrm{H}$, et al. Dose-finding and efficacy confirmation trial of superselective intra-arterial infusion of cisplatin and concomitant radiotherapy for patients with locally advanced maxillary sinus cancer (JCOG1212, RADPLAT-MSC). Jpn J Clin Oncol. 2015;45(1):119-22. doi: 10.1093/jjco/hyu169. [PubMed: 25332419].

25. Homma A, Hayashi R, Matsuura K, Kato K, Kawabata K, Monden $\mathrm{N}$, et al. Lymph node metastasis in $\mathrm{t} 4$ maxillary sinus squamous cell carcinoma: incidence and treatment outcome. Ann Surg Oncol. 2014;21(5):1706-10. doi: 10.1245/s10434-014-3544-6. [PubMed: 24554063].

26. Le Q, Fu KK, Kaplan MJ, Terris DJ, Fee WE, Goffinet DR. Lymph node metastasis in maxillary sinus carcinoma. Int J Radiat Oncol Biol Phys. 2000;46(3):541-9. doi: 10.1016/s0360-3016(99)00453-8.

27. Sugiyama S, Iwai T, Oguri S, Koizumi T, Mitsudo K, Tohnai I. Facial nerve paralysis after super-selective intra-arterial chemotherapy for oral cancer. Int J Oral Maxillofac Surg. 2017;46(6):682-6. doi: 10.1016/j.ijom.2017.01.011. [PubMed: 28209378] 